\title{
Short-Time Behavior and Universality in Irreversible Models
}

\author{
Tânia Tomé \\ Instituto de Física \\ Universidade de São Paulo \\ Caixa Postal 66318 \\ 05315-970 São Paulo, São Paulo, Brazil
}

Received 1 December 1999

\begin{abstract}
We discuss the issue of universality in nonequilibrium phase transitions. We present an introduction to the approach of statistical mechanics that is grounded in the construction and analysis of stochastic lattice models. The concept of irreversibility is studied through the analysis of the condition of detailed balance. The role of symmetry and irreversibility on the critical behavior of the nonequilibrium models is examined. We focus on short-time universal behavior of reversible and irreversible models.
\end{abstract}

\section{Introduction}

Irreversible systems have been treated, mainly, by two distinct statistical mechanical approaches. One of them is grounded on the Zubarev nonequilibrium statistical operator [1, 2]. It generalizes the Gibbs ensembles by taking into account memory effects. The method has been shown to be very appropriate to study the thermodynamical properties of highly excited plasma in semiconductors $[2,3]$. The other approach, used extensively in the last years, is based on the construction and analysis of lattice gas models [4-7] that evolve in time according to stochastic irreversible dynamics, that is dynamics that lacks detailed balance. This approach is suitable to study critical phenomena in nonequilibrium systems. Also, it is used to mimic biological problems and chemical reactions. This is the kind of approach that we consider here.

An important aspect in the study of irreversible as well as reversible systems is the role of symmetry. Distinct systems with the same symmetry are expected to have similar critical behavior. In reversible systems, the symmetry is to be found in the Hamiltonian. In irreversible systems, on the other hand, the symmetry is to be found in the dynamics that defines the model. It is important to note that there are models, such as the contact process [8], which has an absorbing state, that are intrinsically irreversible and do not have symmetries that can be compared to reversible models defined by a Hamiltonian. With respect to the critical behavior, there are at least two kinds of irreversible stochastic models: those for which there exists a reversible model with similar critical behavior and those lacking a re- versible analog.

We present here a discussion about classes of models that include irreversible and reversible models with the same symmetries. We focus our attention on the following statement: models with the same symmetries and defined on the same lattice, reversible or not, are in the same universality class $[4,9,10]$. Numerical calculations of the critical exponent on several models give support to this proposition [11-19].

We also study the short-time universality of reversible and irreversible stochastic lattice models. It was established by Janssen, Schaub and Schmittmann [20], using renormalization group analysis, that there is a universal behavior in the first time steps (after a microscopic time) of the evolution of the system. This behavior has been verified numerically by several studies on the kinetic Ising model and the kinetic Potts model [21-28]. The two models evolve in time according to a dynamics such that in the stationary state the condition of detailed balance is obeyed. That is, they are microscopic reversible models. In addition to these models, short-time universality has also been observed in models without microscopic reversibility [10,29-32]. For example, Ref. [31] considered a probabilistic cellular automaton defined on a square lattice and evolving in time according to an irreversible dynamics with updown symmetry. It was found numerically that both the static and short-time dynamical critical exponents are the same as that of the Ising model defined in the same lattice.

The models that we examine are presented in Section II. There is also a discussion about the role of symmetry and of irreversibility in the study of nonequi- 
librium phase transitions. In Section III we give a brief introduction to short-time analysis and short-time universality, and report some numerical results on reversible and irreversible models. In Section IV we present a summary of our results.

\section{Models}

We treat systems that undergo continuous phase transitions and suppose that the systems are described by models with stochastic dynamics that are either probabilistic cellular automata, or are governed by a master equation. The models are defined on regular lattices; for each site $i$ there is a local variable $\sigma_{i}$, so that the microscopic state of the system is denoted by $\sigma=\left\{\sigma_{i}\right\}$.

The evolution in time of a probabilistic cellular automaton is governed by the equation

$$
P_{\ell+1}(\sigma)=\sum_{\sigma^{\prime}} W\left(\sigma, \sigma^{\prime}\right) P_{\ell}\left(\sigma^{\prime}\right)
$$

where $W\left(\sigma, \sigma^{\prime}\right)$ is the transition probability from $\sigma^{\prime} \rightarrow$ $\sigma$, given by

$$
W\left(\sigma, \sigma^{\prime}\right)=\prod_{i} w\left(\sigma_{i}, \sigma^{\prime}\right)
$$

and $P_{\ell}(\sigma)$ is the probability of state $\sigma$ at time step $\ell$.

Stochastic models with continuous-time evolution are governed by a master equation

$$
\frac{d}{d t} P(\sigma, t)=\sum_{\sigma^{\prime}}\left\{W\left(\sigma, \sigma^{\prime}\right) P\left(\sigma^{\prime}, t\right)-W\left(\sigma^{\prime}, \sigma\right) P(\sigma, t)\right\}
$$

where now $W\left(\sigma, \sigma^{\prime}\right)$ is the transition rate from $\sigma^{\prime}$ to $\sigma$ and $P(\sigma, t)$ is the probability of state $\sigma$ at time $t$. These models are usually called stochastic lattice gas models.

The condition of detailed balance is given by

$$
W\left(\sigma, \sigma^{\prime}\right) P\left(\sigma^{\prime}\right)=W\left(\sigma^{\prime}, \sigma\right) P(\sigma),
$$

where $P(\sigma)$ is the stationary probability. If the model obeys this relation, in the steady state, then it is microscopically reversible, and the stationary states are equilibrium states. If the dynamics is irreversible the system evolves in time and eventually reaches a nonequilibrium steady state where condition (4) is not obeyed. These considerations are valid for probabilistic cellular automata and for stochastic lattice gas models. We remark that if the system is irreversible, then the symmetries are imposed on $W\left(\sigma, \sigma^{\prime}\right)$ and not on the Hamiltonian, as is usual for reversible (equilibrium) models.

If the stochastic model evolves in time according to a dynamics involving only single-spin flips, the master equation is given by

$$
\frac{d}{d t} P(\sigma, t)=\sum_{i}\left\{w_{i}\left(\sigma^{i}\right) P\left(\sigma^{i}, t\right)-w_{i}(\sigma) P(\sigma, t)\right\}
$$

where $\sigma^{i}$ denotes the state obtained from $\sigma$ by flipping the $i$-th spin and $w_{i}(\sigma)$ is the rate of flipping of the $i$-th spin. For the case of the kinetic Ising model and irreversible models with up-down symmetry the flipping rate $w_{i}(\sigma)$ is given by

$$
w_{i}(\sigma)=\frac{1}{2}\left\{1-\sigma_{i} f_{i}(\sigma)\right\}
$$

where $\sigma_{i}= \pm 1$ is an Ising variable and $f_{i}(\sigma)$ is an odd function of the nearest-neighbor spins of the $i$-th spin. Glauber dynamics [33], whose stationary state gives the static properties of the nearest-neighbor Ising model defined in a regular lattice, is such that

$$
f_{i}(\sigma)=\tanh \left(K \Sigma_{\delta} \sigma_{i+\delta}\right)
$$

where the summation is over the nearest neighbors of site $i$. In addition to the Glauber prescription, there are other stochastic dynamics used in computer simulations of the Ising model. One example, very well known, is the Metropolis prescription [34].

We can also define the majority vote model which is a very simple example of an irreversible model with up-down symmetry. In this case we have

$$
f_{i}(\sigma)=(2 p-1) g\left(\Sigma_{\delta} \sigma_{i+\delta}\right)
$$

where the summation is over the nearest neighbors of the central site $\sigma_{i}$. The function $g(X)$ assumes the value 1 if $X$ is greater than zero, 0 if $X$ is equal to zero and -1 if $X$ is less than zero. So if a site $i$ is randomly chosen and the majority of its neighbors are in state 1 then in the next instant of time the dynamical variable $\sigma_{i}$ will assume the value 1 with probability $p$ and -1 (contrary to the majority) with probability $q=1-p$. It is straightforward to verify that the flipping rate $(6)$ with $f_{i}(\sigma)$ given in $(8)$ possesses up-down symmetry, that is, it is invariant under the inversion $\sigma_{i} \rightarrow-\sigma_{i}$ for all $i$. Mean-field analysis [35] and results from Monte Carlo simulations [13] show that the model presents three phases: paramagnetic, ferromagnetic and antiferromagnetic. The ferromagnetic state is stable for values of $q$ less than a critical value $q_{c}$, where there is a continuous phase transition. It was also been verified [13] from Monte Carlo simulations that the majority vote model defined on a square lattice has the same critical behavior, with respect to the static critical exponents, as the Ising model defined in the same lattice. It was found that $\beta / \nu=0.125 \pm 0.005$ and $\gamma / \nu=1.73 \pm 0.05$, which are in good agreement with the expected results for the Ising model in two dimensions, namely, $\beta / \nu=1 / 8=0.125$, and $\gamma / \nu=7 / 4=1.75$. This is convincing evidence that models without microscopic reversibility, but possessing up-down symmetry, are in the same universality class as the Ising model.

It is possible to see explicitly that the majority vote model, defined above, does not obey condition (4). 
Consider a sequence of microscopic states where spins are flipped in consecutive time steps in such a way that we depart from an initial state and at the end we come back to this same state. Start, for example, from the following cluster in a configuration $A$

$$
\begin{array}{rrrr}
+ & + & \\
+ & + & - & + \\
+ & - &
\end{array}
$$

Flipping one of the central sites we can have the following situation $B$ in the next instant of time

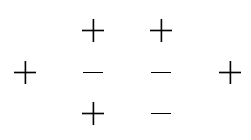

In the next instant of time, we can have the configuration $C$,

$$
\begin{array}{rrr}
+ & + & \\
+ & - & + \\
+ & -
\end{array}
$$

and in the next instant of time, the configuration $D$

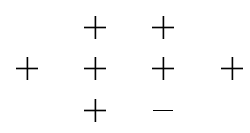

From state $D$ we can return to the initial state $A$. For the cyclic sequence $A \rightarrow B \rightarrow C \rightarrow D \rightarrow A$ we obtain a transition rate that equals $\frac{1}{2} p q^{2}$ and for the reverse transformation $A \rightarrow D \rightarrow C \rightarrow B \rightarrow A$ the transition rate equals $\frac{1}{2} p^{2} q$. These two transition rates are different, which proves that the model is indeed irreversible. The ferromagnetic, antiferromagnetic and paramagnetic states that occur in the model are nonequilibrium steady states, and it is not possible to associate, a priori, a Gibbs distribution for these states.

If we perform the same kind of calculation for the Glauber dynamics, with $f_{i}(\sigma)$ given by equation ( 7$)$, it is easy to see that the transition rates for any cyclic transformation and the reverse transformation are the same. In particular, for the sequences $A \rightarrow B \rightarrow C \rightarrow$ $D \rightarrow A$ and $A \rightarrow D \rightarrow C \rightarrow B \rightarrow A$, both transition rates equal $\left(\frac{1}{2}\right)^{4}(1+\tanh 2 K)^{2}(1+\tanh 4 K)$. Actually, it is straightforward to prove detailed balance (4) for the Glauber prescription.

Other examples of reversible and irreversible models with the same symmetries are the kinetic three-state Potts model and a probabilistic cellular automaton with $C_{3 v}$ symmetry [10]. In the case of the automaton, we consider a regular square lattice with $N$ sites. To each site is associated a variable $\sigma_{i}$ that takes the values 1,2 , and 3 . The transition probability depends only on the values of the four nearest neighbor sites of the central site $i$. The dynamics follows rules that are similar to the ones of the majority vote model. If there is majority of neighbors in one of the three states then the central site changes to the majority state with probability $p$ and to one of the other two states with probability $(1-p) / 2$. If there is no majority, then the central site assumes one of the three possible states with probability $1 / 3$. It is straightforward to check that the transition probability so defined is invariant under the symmetry operations of the group $C_{3 v}$. That is, the rotation symmetry operations $1 \rightarrow 2 \rightarrow 3 \rightarrow 1$ and $1 \rightarrow 3 \rightarrow 2 \rightarrow 1$ and the specular operations $1 \longleftrightarrow 2,2 \longrightarrow 3$, and $1 \longrightarrow 3$ leave the transition rate invariant. Numerical simulations show that the system exhibits a continuous phase transition with the ordered state occurring at high values of $p$. As $p$ is decreased the transition takes place at a critical value $p_{c}$ and the system becomes disordered for $p<p_{c}$. Using finite-size scaling the critical exponents were estimated as $\beta / \nu=0.134 \pm 0.005$ and $\gamma / \nu=1.74 \pm 0.02$ [10]. These values are in fair agreement with the results for the three state Potts model in two dimensions, namely, $\beta / \nu=2 / 15=0.133 \ldots, \gamma / \nu=26 / 15=1.733 \ldots$

\section{Short-time universal be- havior}

Consider a model defined on a lattice and evolving in time according to a given stochastic dynamics, for example, a kinetic Ising model with Glauber dynamics. We prepare the system in an random initial configuration and observe what happens when we let the system evolve in time, by means of a Monte Carlo simulation. The main idea of the short-time universality theory is that if the system is placed at the critical temperature and we analyze its behavior in the very beginning of the simulation it is also possible to find universality. For example the magnetization increases in time according to a power law with a new dynamic critical exponent $\theta$. This universal regime sets in after a microscopic time. Although the mechanism responsible, at the microscopic level, for the initial increase of the magnetization is not yet very well understood, Janssen, Schaub and Schmittman [20] determined the scaling relations governing short-time behavior. Their statements are based on renormalization group analysis. The short-time scaling theory predicts the following critical behavior

$$
M(t) \sim t^{\theta}
$$

and

$$
M_{2}(t) \sim t^{\zeta}
$$

for the magnetization $M(t)$ and for the second moment of the magnetization $M_{2}(t)$, where $\zeta=(d-2 \beta / \nu) / z$ with $d$ the lattice dimension and $z$ the dynamic critical exponent. The static critical exponents $\beta$ and $\nu$ are related to the magnetization and correlation length.

The exponent $\theta$ has been evaluated numerically by several authors for the kinetic Ising model and for the kinetic Potts model. Different initial conditions and 
different reversible dynamics for the Ising model in two dimensions lead to the same universal result $\theta=0.19$ [22] [23, 26, 30].

More recently the short-time behavior of irreversible stochastic models (without detailed balance), has also been investigated [29-32]. In this case one studies irreversible models that are far from the stationary nonequilibrium state, and observes the behavior of the order parameter in the first steps of a numerical simulation. The parameters that define the irreversible dynamics are set right at their critical values. Of course, it is difficult to prepare the irreversible system with the critical parameters since the parameters themselves must be also found from numerical simulations. But there are irreversible systems for which the regime where the magnetization increases as a power law with exponent $\theta$ takes place right after the first Monte Carlo steps [29, 30]. The value of $\theta$ is extracted easily from a log-log plot of the magnetization versus time $t$. Irreversible systems with up-down symmetry in two dimensions have the same universal exponent $\theta$ as the two dimensional kinetic Ising model. For example, calculations on the majority vote model and on the kinetic Ising model with Glauber dynamics give $\theta=0.190 \pm 0.005$ and $\theta=0.191 \pm 0.002$, respectively [30]. So the exponent $\theta$ for the irreversible model and for the reversible model are in good agreement. The same behavior is observed for other irreversible stochastic lattice gas models with up-down symmetry [30]. Other dynamic exponents associated to the short-time regime, such as the exponent for the second moment of the magnetization, are also consistent for reversible and irreversible models with up-down symmetry.

These results were obtained by starting from a number of initial independent configurations. Each initial configuration was generated by setting the spin of each site up or down with equal probability, independently. Then the system evolves according to the local rules of the model. The magnetization and its second moment are measured at each Monte Carlo step. Finally, averages are performed, at each time step, over the set of independent initial configurations. The analysis of the time evolution of the magnetization and the second moment of the magnetization, in the early-time regime, give the universal exponents $\theta$ and $\zeta=(d-2 \beta / \nu) / z$. To obtain these exponents from numerical simulations we use the short-time scaling relations (9) and (10).

Other dynamic exponents as, for example, the exponent $z$ can be evaluated from the short-time analysis. To do this it is necessary to calculate the fourth moment $M_{4}(t)$ of the magnetization, from which we get the time-dependent Binder cumulant $U=1-M_{4} /\left(3 M_{2}^{2}\right)$. The following scaling relation for the Binder cumulant at the critical point holds

$$
U(t, L)=\phi\left(t / L^{z}\right),
$$

where $L$ is the linear size of the lattice. Some works [22]
[29] determine the critical exponent $z$ from this relation, via a data-collapse analysis for lattices of different size $L$. However, these results are not very accurate since the collapse can present inherent errors.

For the case of the three-state kinetic Potts model the value of $\theta$ is estimated as $\theta=0.08$ [26]. Calculations with the irreversible probabilistic cellular automaton with the same symmetries of the three-state Potts, defined in Section II, give $\theta=0.09$ [10]. To analyze and compare these values of $\theta$ we have to take into account the fact that calculations of the short-time behavior for probabilistic cellular automata are more intricate than calculations using models with continuous-time dynamics. Besides, it is known that the short-time behavior is more difficult to obtain for models with three states per site, such as the kinetic three-state Potts model. So we can say that on a first approach, the estimated values of $\theta$ are in agreement. More calculations must be done on both the reversible and the irreversible models, to obtain more precise values for the dynamical critical exponents.

\section{Summary}

We reviewed some aspects of nonequilibrium phase transitions that have been studied in the last years. We presented the approach that we use to study critical behavior in irreversible stochastic models. The concept of microscopic reversibility was discussed by taking into account the condition of detailed balance. We also show that it is possible to check whether a model is microscopically reversible by calculating the rate of transition for a closed path in the space of configurations. With this definition in hand we discussed some aspects concerning universality in nonequilibrium phase transitions. Our studies indicate that an irreversible model, defined by a stochastic dynamics, and a reversible model, defined by a Hamiltonian, belong to the same universality class if they possess the same symmetries and are defined on the same lattice. This result was proposed first by Grinstein and collaborators [4] for systems with up-down symmetry. We proposed that irreversible models with $C_{3 v}$ symmetry are in the same universality class as the three-state Potts model.

In contrast to the above mentioned models, there are nonequilibrium models with absorbing states, which do not belong to the universality class of any equilibrium model. It is worth saying that the continuous phase transitions in models with one absorbing state are related to the directed percolation problem [36].

The universality in nonequilibrium phase transitions was also viewed in the context of short-time behavior. We briefly reviewed the main ideas concerning this new universal regime, and discussed results obtained for models with up-down symmetry and models with $C_{3 v}$ symmetry. Again, we conclude that the short-time 
universal behavior is the same for models possessing the same symmetries and defined on the same lattice.

Symmetry is, perhaps, the very first idea that we can have to study phenomena that occur in nature. It is well known that symmetry is the most important feature in the Landau theory of phase transitions. It also plays a fundamental role in the classification of critical behavior into universality classes.

\section{Acknowledgments}

I thank J. R. Drugowich de Felício for useful discussions.

\section{References}

[1] D. N. Zubarev, Nonequilibrium Statistical Thermodynamics (Consultants Bureau, New York, 1974).

[2] A. R. Vasconcellos, R. Luzzi and L. S. Garcia-Colin, Phy. Rev. A 43, 6622 (1991).

[3] T. Tomé, A. R. Vasconcellos and R. Luzzi, Physica B 144, 376 (1987).

[4] G. Grinstein, C. Jayaprakash and Y. He, Phys. Rev. Lett. 55, 2527 (1985).

[5] R. Dickman, Phys, Lett A, 122, 463 (1987).

[6] T. Tomé, Irreversibilidade, (IFUSP, São Paulo, Brazil, 1996).

[7] J. Marro and R. Dickman, Nonequilibrium Phase Transitions in Lattice Models (Cambridge University Press, Cambridge, 1999).

[8] T. M. Liggett, Interacting Particle Systems (SpringerVerlag, New York, 1885).

[9] T. Tomé, A. Brunstein and M. J. de Oliveira, submitted to publication.

[10] A. Brunstein and T. Tomé, Phys. Rev. E 60, 3666 (1999).

[11] H. W. J. Blöte, J. R. Heringa, A. Hoogland an R. K. P. Zia, J. Phys. A 23, 3799 (1990).

[12] H. W. J. Blöte, J. R. Heringa, A. Hoogland an R. K. P. Zia, Int. J. Mod. Phys. B 5, 685 (1990).

[13] M. J. de Oliveira, J. Stat. Phys. 66, 273 (1992).

[14] M. J. de Oliveira, J. F. F. Mendes and M. A. Santos, J. Phys. A 26, 2317 (1993).
[15] P. Tamayo, F. J. Alexander and R. Gupta, Phys. Rev. E 50, 3474 (1995).

[16] M. A. Santos and S. Teixeira, J. Stat. Phys. 78, 963 (1995).

[17] T. Tomé and J. R. Drugowich de Felício, Phys. Rev. E 53, 3976 (1996).

[18] N. R. S. Ortega, C. F. Pinheiro, T. Tomé and J. R. Drugowich de Felício, Physica A 255, 189 (1998).

[19] A. Brunstein and T. Tomé, Physica A 257, 334 (1998).

[20] H. K. Janssen, B. Schaub and B. Schmittmann, Z. Phys. B 73, 539 (1989).

[21] D. A. Huse, Phys. Rev. B 40, 304 (1989).

[22] Z. B. Li, U. Ritschel and B. Zheng, J. Phys. A 27, L837 (1994).

[23] Z. B. Li, L. Schülke and B. Zheng, Phys. Rev. Lett. 74, 3396 (1995).

[24] Z. B. Li, L. Schülke and B. Zheng, Phys. Rev. E 53, 2940 (1996).

[25] U. Ritschel and P. Czener, Phys. Rev. E 55, 3958 (1997).

[26] K. Okano, L. Schülke, K. Yamagishi and B. Zheng, Nuclear Physics B 485, 727 (1997).

[27] L. Schülke and B. Zheng, Phys. Lett. A 204, 295 (1995).

[28] B. Zheng, Phys. Rev. Lett. 77, 679 (1996).

[29] J. F. F. Mendes and M. A. Santos, Phys. Rev. E 57, 108 (1998).

[30] T. Tomé and M. J. de Oliveira, Phys. Rev E 58, 4242 (1988).

[31] T. Tomé and J. R. Drugowich de Felício, Mod. Phys. Lett B 12, 873 (1998).

[32] A. Brunstein and T. Tomé, Mod. Phys. Lett. B 13, 471 (1999).

[33] R. J. Glauber, J. Math. Phys. 4, 294 (1963).

[34] N. Metropolis, A. W. Rosenbluth, A. H. Teller, J. Chem. Pys. 21, 1087 (1953).

[35] T. Tomé, M. J. de Oliveira and M. A. Santos, J. Phys. A 24, 3677 (1990).

[36] P. Grassberger, Z. Phys. B 47, 365 (1982). 